\title{
A comparative analysis on question classification task based on deep learning approaches
}

\author{
Muhammad Zulqarnain ${ }^{\text {Corresp., }}{ }^{\text {, }}$ Ahmed Khalaf Zager Alsaedi ${ }^{2}$, Rozaida Ghazali $^{1}$, Muhammad Ghulam Ghouse ${ }^{1}$, \\ Wareesa Sharif $^{3}$, Noor Aida Husaini ${ }^{1}$ \\ ${ }^{1}$ Faculty of Computer Science and Information Technology, Universiti Tun Hussein Onn Malaysia (UTHM), BATU PAHAT, JOHOR, Malaysia \\ 2 Physic Department, College of Science, College of Science, University of Misan, Iraq, Iraq \\ 3 Faculty of Computing, The Islamia University Bahawalpur, Bahawalpur, Punjab, Pakistan \\ Corresponding Author: Muhammad Zulqarnain
}

Email address: hi160052@siswa.uthm.edu.my

Question classification is one of the essential tasks for automatic question answering implementation in natural language processing (NLP). Recently, there are several textmining issues such as text classification, document categorization, web mining, sentiment analysis, and spam filtering that has been successfully achieved by deep learning approaches. In this study, we illustrated and investigated our work on certain deep learning approaches for question classification tasks in an extremely inflected Turkish language. In this study, we trained and tested the deep learning architectures on the questions dataset in Turkish. In addition to this, we used three main deep learning approaches; Gated Recurrent Unit (GRU), Long Short-Term Memory (LSTM), Convolutional Neural Networks (CNN), and we also applied two different deep learning combinations of CNN-GRU and CNN-LSTM architectures. Furthermore, we applied the Word2vec technique with both skip-gram and CBOW methods for word embedding with various vector sizes on a large corpus composed of user questions. By comparing analysis, we conducted an experiment on deep learning architectures based on test and 10-cross fold validation accuracy. Experiment results were obtained to illustrate the effectiveness of various Word2vec techniques that have a considerable impact on the accuracy rate using different deep learning approaches. We attained an accuracy of $93.7 \%$ by using these techniques on the question dataset. 


\section{A comparative analysis on question classification task based on deep learning approaches}

Muhammad Zulqarnain ${ }^{1}$, Ahmed Khalaf Zager Alsaedi², Rozaida Ghazali ${ }^{1}$, Muhammad Ghulam Ghouse $^{1}$, Wareesa Sharif ${ }^{3}$, Noor Aida Husaini ${ }^{1}$

${ }^{1}$ Department of Computer Science and Information Technology, Universiti Tun Hussein Onn Malaysia, Batu Pahat, Johor, Malaysia.

${ }^{2}$ Physic Department, College of Science, University of Misan, Ministry of Higher Education of Iraq

${ }^{3}$ Faculty of Computing, The Islamia University of Bahawalpur, Bahawalpur, Punjab, Pakistan Corresponding Author:

Muhammad Zulqarnain ${ }^{1}$

Lorong Haji Hassan, Parit Raja, Johor, 86400, Malaysia

Email address: zulqarnainmalik321@email.com

\section{ABSTRACT}

Question classification is one of the essential tasks for automatic question answering implementation in natural language processing (NLP). Recently, there are several text-mining issues such as text classification, document categorization, web mining, sentiment analysis, and spams filtering that have been successfully achieved by deep learning approaches. In this study, we illustrated and investigated our work on certain deep learning approaches for question classification tasks in an extremely inflected Turkish language. In this study, we trained and tested the deep learning architectures on the questions dataset in Turkish. In addition to this, we used three main deep learning approaches; Gated Recurrent Unit (GRU), Long Short-Term Memory (LSTM), Convolutional Neural Networks (CNN), and we also applied two different deep learning combinations of CNN-GRU and CNN-LSTM architectures. Furthermore, we applied the Word2vec technique with both skip-gram and CBOW methods for word embedding with various vector sizes on a large corpus composed of user questions. By comparing analysis, we conducted an experiment on deep learning architectures based on test and 10-cross fold validation accuracy. Experiment results were obtained to illustrate the effectiveness of various Word2vec techniques that have a considerable impact on the accuracy rate using various deep learning approaches. We attained an accuracy of $93.7 \%$ by using these techniques on the question dataset.

Subjects Artificial Intelligence, Data Mining, and Deep Learning

Keywords Convolutional neural networks, Long short term memory, Gated recurrent unit, Word2vec, Question classification 
41

42

43

44

45

46

47

48

49

50

51

52

53

54

55

56

57

58

59

60

61

62

63

64

65

66

67

68

69

70

71

72

73

74

75

76

77

78

79

\section{INTRODUCTION}

With the rapid development of computer technology and the internet, a huge amount of textual data in digital form are generated every day (Wang and Qu, 2017), and retrieve the given contents from a large amount of information rapidly and accurately. This has become an ordinary issue. Textual data is highly dimensional data having, comprising, consisting of irrelevant and unwanted features that are difficult to manage and maintain (Sharif et al., 2017). In NLP, the role of the question classification system is to predict the form of precise response according to the query. However, in most of the cases in the NLP, what the user desires to ask of the right answer to the question individually. One of the most appealing areas is the question answering (QA) system for both company and organization which provides more appropriate access to information than the conventional search engine. Question Answering (QA) is one of the main technologies in a QA system that automatically seeks correct answers in natural language to random questions. For the information retrieval, the QA systems have been successfully applied in NLP and achieved significant results.

Furthermore, the implementation of a question classification system is identifying the category of sort questions that are asked in NLP. QA system usually consists of four steps, semantic understanding, question classification, text retrieval, and answer extraction (Le, Phan \& Nguyen, 2015). Most of the useful step is a question classification which can give useful information for subsequent execution and involves the customer category answer, the intent of question classification, and so on. The correlation between the questions and the category can be illustrated through a corresponding process:

$$
F: X=\left\{C_{1}, C_{2}, \ldots, C_{n}\right\}
$$

Where Turkish questions are represented by $X$, while $\left\{C_{1}, C_{2}, \ldots, C_{n}\right\}$ refers the set of categories, and $F$ defines the question, $X$ is classified into a specific category $C_{i}$ through some rules. In the significant part of the QA system, there are two key dialog implementations to determine the questions to the credible classification of the answers (Mohd \& Hashmy, 2018). Identifying the questions is one of the defined matters according to the nature of the question. For instance, the question about the comparison is that "What is the difference between music and noisiness?" After categories, the questions of the QA system can perform the subsequent execution to improve the accuracy of the answer according to their intents. The other way of dialog implementation is to identify the questions based on the user requirement. For example, the question "Who is the chief of army staff of Malaysia?" is a question about a character or is a type of human (person). As per the question categorization, the QA system should usage the search technique specific to the human (person) type. Consequently, more efficient question identification can enhance the performance of the QA system.

Question classification is one of the correlated problems to documents categorization (Ehsan \& Mojgan, 2014). However, in recent times, document categorization has been provided a huge 
80 quantity of scientific contribution. While question classification is mainly in the Turkish 81 language now is a novel academic issue. The most important difference between question 82 classification and document categorization is that the document dimension is much longer than 83 the question dimension. Therefore, each character and word in question classification could be 84 meaningful. As a results, generating features from a single question is more difficult than 85 generating features from a large text (Ehsan \& Mojgan, 2014). There are various approaches; 86 machine learning, rule-based and hybrid approaches (Razzaghnoori, Sajedi \& Reghuraj, 2018) 87 have been used in the question classification tasks. In our research, some deep learning 88 approaches such as LSTM, GRU, CNN, and their combination are utilized to classify user 89 questions based on Word2vec techniques both Continue Bag of Words (CBOW) and skip gram. 90 In this study, our main contributions are: most articles associated to question classification 91 concentrates on the English language and they have not studied an agglutinative language where the structure of words is generated by putting suffixes (morphemes) to the root of the word. The Turkish language has some distinctive features, which makes it problematic and has been demonstrated challenging for NLP. The majority of the challenges drives from Turkish complex morphology and how it deals with syntax.

Turkish, as a non-indo-European language, has several unique features, that make NLP difficult to determine. For example, the Turkish language does not have grammatical gender and noun classes (Le, Phan \& Nguyen, 2015). In particular, it is very difficult to extract these nuances by NLP techniques, which have been generally applied for Indo European languages like English and German. Moreover, natural languages have several words that come from the same morphological class for structural and grammatical purposes. Particularly, in Turkish language, there are large number of word formations due to language structure. Turkish language uses the derivative affixes and the inflectional to construct new words that typically produce a few hundred structures, and each verbal root can produce a million structures (Ozturkmenoglu \& Alpkocak, 2012). For this reason, words that mean nearly a sentence in English language are more probable to be derived. When used contexts in a sentence, the Turkish words may provide a several derivational and inflectional suffixes for example;

$$
\begin{aligned}
& \text { dün }+ \text {-di }+ \text { biz }+ \text { içinde }+ \text { ofis } \rightarrow \rightarrow \text { yesterday, we were in the office. } \\
& \text { gor }+ \text { onlar }+ \text { görmek }+ \text { yapabilmek }+\mathrm{o} \rightarrow \rightarrow \text { they were able to see it. }
\end{aligned}
$$$$
\text { İngiltere }+ \text { başbakanı }+\mathrm{kim} \rightarrow \longrightarrow \text { who is the prime minister of England? }
$$

For this purpose, in most situations, the lemmatization process is very significant for obtaining the uninflected word forms in order to use IR (information retrieval) and other text processing techniques to tackle Turkish. The lemmatization method is commonly employed to enhance the efficiency of information retrieval systems. The main goal of lemmatization is to reduce inflectional forms and sometimes derivationally related forms of words to root forms. It establishes a connection between surface form of connected words and dictionary forms. For above-mentioned reasons, in contrast to the English language, there is no useful lemmatization 
120

121

122

123

124

125

126

127

128

129

130

131

132

133

134

135

136

137

138

139

140

141

142

143

144

145

146

147

148

149

150

151

152

153

154

155

156

157

158

159

method in Turkish, due to the language structure of Turkish. This is another issue with Turkish language when studying text processing (for more details about the difficulty of Turkish see (Oflazer; 2014)).

Another issue with the Turkish language is that it may neglect the tools required to determine the text information. However, we could not find any Turkish question datasets at the start of this study, so we decided to convert an English question dataset into a Turkish question dataset to see how the proposed approaches performed well in their best way.

On the other hand, Mikolov et al.(2013) introduced a new method of feature representation, which is applied in the feature extraction step is known as distributed representations of words or Word2vec (Mikolov et al., 2013). The concept behind the word representation approach is the terms with a semantic or syntactic connection which is used with higher probability in a similar context (Liu et al., 2017). Therefore, the vectors of those words need a little bit close to each other if word 1 and word 2 contain similar contexts.

The learning algorithms of Word2vec are representing the words in a vector space and achieve superior results in the NLP mechanism to identify the relevant words. In the case of distributed representation of words, the neural network is very extraordinary to compute vector code of several linguistic regularities and patterns (Mikolov et al., 2013). In some cases, most models can be illustrated as linear representations instantaneously. For instance, the outcome of a vector computation vec('king') — vec(“'man') ? vec("women') is closer to vec("queen') than to any other word vectors (Mikolov et al., 2013).

The main purpose of our research is a comparative analysis between the deep learning architecture and Word2vec method. Therefore, the major contributions of this study are presented as follows:

- Most articles focused on the English language have associated with question classification and they have not worked an agglutinative language where the construction of words is produced by assigning suffixes (morphemes,) to the root of the words. In NLP, the Turkish language has proved the preprocessing problem. As a non-Indo-European language, there are several unique features in Turkish languages that make NLP challenging.

- Another contribution is the impact of employing various Word2vec pre-trained word embeddings on various deep learning approaches. In our study, the first approach presented was to use Word2 vec methods that are Continuous Bag of Words, skip gram to cluster words in the corpus and convert all words into vectors in the space. For the extraction of word vectors, the Word2vec method is applied to extract as a variation of the query word vectors of words. After that, the deep learning approaches such as CNN, GRU, LSTM, and their combinations including CNN-LSTM and CNN-GRU are applied for question classification. By using these four various approaches, the average correctness of CNN is $92.46 \%$, LSTM achieved 90.89\%, GRU obtained 91\%, CNN-LSTM and CNN-GRU got 91.7\% and 92.36\% respectively over the Turkish question dataset.

- Moreover, there was no Turkish question labeled dataset as well, so in this study, we added a new Turkish question dataset which is translated from UIUC English question dataset.

PeerJ Comput. Sci. reviewing PDF | (CS-2020:10:54597:2:0:NEW 27 Apr 2021) 
160

161

162

163

164

165

166

167

168

169

170

171

172

173

174

175

176

177

178

179

180

181

182

183

184

185

186

187

188

189

190

191

192

193

194

195

196

197

198

199

\section{RELATED WORK}

Question answering classification is an essential task of text classification. In the early 1950s, IBM was provided an environment to leading in examining text identification. Later, in the 1960s, Marun and Kahns introduced the keyword technique in the texts to automatically categories the chosen texts.

There are three individual stages in the classic question answering system (Ehrentraut et al., 2018):

1. Question processing: this is an initial stage in questioning and answering systems where questions are asked by users (Madabushi, Lee \& Barnden, 2018). The aim of this stage is understood to apply the logical calculations for the representation and categorization of the questions.

2. Extraction and processing of documents: a set of relevant documents are selected in this stage and a set of paragraphs are captured which depend on the concentrations of the issue.

3. Answer processing: The purpose of this stage is considered to respond based on the relevant fragments of the documents. The preprocessing of the data requires pairing an answer based upon the similar contexts of the question asked. The general architecture of natural language for the question answering system is presented in Fig1.png, (Athira, Sreeja \& Reghuraj, 2013).

Several different approaches have been successfully used in the question classification issue. Most of these approaches are divided into four groups: rule-based approaches, machine learning approaches, deep learning approaches, and hybrid approaches (Razzaghnoori, Sajedi \& Reghuraj, 2018; Hao, Xie \& Xu, 2015). Author Galitsky worked on rule-based approaches to classify questions based on the pair of questions with manually written rules according to the provided contents (Galitsky; 2017). While determining the particular rules is a massive time and struggle to process a variety of questions. However, the deep learning approaches, machine learning approaches, and rule-based techniques are capable to automatically construct a precise classification implementation utilizing different features of questions (Ehsan \& Mojgan, 2014).

The machine learning techniques are superior to manual techniques discussed by authors (Sarrouti \& Alaoui, 2017). They described machine learning techniques give a reasonably easy way to classify questions as compared to manual techniques. Therefore, with this kind of implementation, the system can learn easily from the data and can be customized to a new system. On the other hand, there are limited studies that have used the hybrid approaches for the classification of the question. Here, we discuss a little bit about some research. The author (Ehsan \& Mojgan, 2014) introduced a hybrid approach for a Persian closed area of question classification system, in which researchers willing a dataset that consists of 9500 questions with the help of some researchers. They achieved a reasonable performance with an accuracy of $80.5 \%$ based on a large number of question classes.

Moreover, to resolve the question classification issues, there are several different machine learning approaches such as Neural Network, Random Forest, SVM, Decision Trees, Naive 
200 Base, KNN that have been applied for classifications. However, for the question classification 201 task in NLP, the SVM ("Support Vector Machine") considers the key approach in machine 202 learning (Sherkat \& Farhoodi, 2014). To obtain their objectives, the authors Sherkat and 203 Farhoodi (Sherkat \& Farhoodi, 2014) used the SVM and dimension reduction method which uses 204 a few linguistic features with a bag of the n-grams feature vector. Similarly, (Huang et al., 2017) 205 applied a tree kernel with an SVM for identification the questions answering and successfully 206 achieved an accuracy of $87.4 \%$ statistics. But during the experiment they did not use semantic 207 and syntactic features. In the same way, the integrated approach is known as Hierarchical 208 Directed Acyclic Graph (HDAG) with kernel function implemented by (Chitra \& Kalpana, 2013) 209 the kernel function as known Hierarchical Directed Acyclic Graph (HDAG) which squarely 210 perform some levels of chunks and their relatives on organized natural language database. Also, 211 on the question answering corpus, $\mathrm{Xu}$ et al., was introduced a hierarchical technique based on the 212 SNoW learning algorithm for the classification of the questions (Xu et al., 2019). In their 213 research, they used a two-phase classification process. In the first phase, they presented the four 214 most potential coarse-grained questions, classes. In the second phase, the question is classified 215 into one of the child classes of the four coarse-grained question classes with an accuracy of $216 \quad 84.2 \%$.

217 Furthermore, the author (Merchant \& Pande, 2018) used the features roughly technique as a 218 similar introduced by the authors of (Mohd \& Hashmy, 2018). Even though the enhancement 219 they applied a dimensionality reduction method that is near to Principal Component Analysis 220 (PCA) also known as Latent Semantic Analysis (LSA) to decrease the feature space for accurate 221 classification. In their study, Back-Propagation Neural Networks (BPNN) and SVM are used. 222 Their study presents that BPNN achieve superior results than SVM. On the other hand, to cluster 223 224

225

226

227

228

229

230

231

232

233

234

235

236

237

238 words in the vocabulary (Razzaghnoori, Sajedi \& Reghuraj, 2018) referred to some clustering algorithm in which perform to convert every question into a vector space. After that, MultiLayered Perceptron (MLP) and SVM were used for the classification of the question. By evaluating the performance of such approaches, they obtain a reasonable accuracy of $73 \%$ using SVM and an accuracy of $72.52 \%$ by using MLP on 3 various datasets. They also prepared the UTQD-2016 dataset ("University of Tehran Question Dataset 2016"). In this corpus, many different types of questions taken from the jeopardy game are shown on official Iran's TV. In their third approach, they used the Word2vec method to convert each question into a matrix where every row presents a Word2vec representation of a word. After that, the authors used an LSTM (Razzaghnoori, Sajedi \& Reghuraj, 2018) approach to classify the questions and reported $81.77 \%$ accuracy on three-question databases. The detailed summary of the related research in question classification is illustrated in Table1.doc.

\section{DEEP LEARNING APPROACHES}

Deep learning approaches were derived from artificial neural networks and nowadays it is a principal area of machine learning and has successfully been applied to achieve excellent 
239

240

241

242

243

244

245

246

247

248

249

250

251

252

253

254

255

256

257

258

259

260

261

262

263

264

265

266

267

268

269

270

271

272

273

274

275

performance in various research areas. However, in the section, we evaluated four types of deep learning models for solving questions answering classification issues.

\section{Convolution Neural Network (CNN)}

The Convolution neural networks are one of the most extensively used models in the deep learning community. And it is the same as a multi-layered perceptron, but it is effectively trained to combine sparsely attached convolutional layers with the completely connected dense layer. CNN consists of three major layers as convolution layer, Max-pooling layer, and fully connected layer for question classification are presented in Fig2.png. However, CNN utilizes only a single layer with variant functions to transfer information from one volume of activation to the next. The Convolutional layer is an essential layer of a CNN model that performs several computational processes. The max-pooling layer, in CNN, performs on data to compress and make it smooth. While for selecting the maximum value of the responsive area the Max-layer is used which produces data-invariant small translational changes (Zulqarnain, Ghazali \& Hassim, 2019). A fully connected layer is used as a final layer of CNN which produces the output by connecting all neurons in the forward and backward manner.

\section{Long Short Term Memory (LSTM)}

A Long Short Term Memory (LSTM) unit is a type of traditional RNN. It was initially introduced by German researchers Sepp Hochreiter and Juergen in 1997 (Hocheriter \& Schmidhuber; 1997). LSTM approach is a variant of the traditional RNN that can learn long sequential data and maintain the propagation of error through all layers (Samarawickrama \& Fermando, 2018). The LSTM contains special internal memory blocks and a gated mechanism that helps to solve the two popular drawbacks which are related to vanishing gradients or exploding in the conventional RNN. In LSTM the memory blocks consist of memory cells with self-connections and particular multiplicative units to handle the flow of information. An LSTM block consists of three gates including input gate, output gate, and forget gate (Lee, 2015). The architecture of the standard LSTM gates block is presented in Fig3.png.

In Fig3.png, the $\left(i_{t}\right),\left(o_{t}\right)$, and $\left(f_{t}\right)$, are the input, output, and forget gates of LSTM through the time step $t$ respectively, $\left(c_{t}\right)$ denoted the memory cell content, $\hat{a}_{t}$ is the candidate state calculated in equation (5). $x_{t}, h_{t}$, and $h_{t-1}$ is the input, final output of the LSTM, and a previous time step of the hidden unit. Update the cell state vector is calculated as in equation (6). To perform the hidden state $\left(h_{t}\right)$ of an LSTM unit that is passed to the next sample in a sequence, the output of the output gate $o_{t}$, equation (3) is multiplied by the squashed cell state $c_{t}$ through tanh function in equation (7), where $\mathrm{W}_{\mathrm{xo}}$ and $\mathrm{U}_{\mathrm{ho}}$ are weight matrix, $\mathrm{b}_{\mathrm{o}}$ is a bias term, and Sigm ( $\left.\mathrm{x}\right)=$ $\frac{1}{1+\mathrm{e}^{-\mathrm{x}}}$.

$$
1+\mathrm{e}^{-1}
$$

$$
i_{t}=\operatorname{Sigm}\left(W_{x i} x_{t}+U_{h i} h_{t-1}+b_{i}\right)
$$


276

277

278

279

280

281

282

283

284

285

286

287

288

289

290

291

292

293

294

295

296

297

298

299

300

301

302

303

$$
o_{t}=\operatorname{Sigm}\left(W_{x o} x_{t}+U_{h o} h_{t-1}+b_{o}\right)
$$

The weights and bias computed during the training process are $W_{i}, W_{o}, W_{f}, W_{\hat{a}} \in R^{m \times p}, U_{i}, U_{o}$ $, U_{f}, U_{\hat{a}} \in R^{m \times m}, b_{i}, b_{o}, b_{f}, b_{\hat{a}} \in R{ }^{m \times 1}$. * is element-wise multiplication of two vectors. Here 'Sigm' is an element-wise logistic sigmoid activation function and 'tanh' is an element-wise hyperbolic tangent activation function.

\section{Gated Recurrent Unit (GRU)}

The GRU is an advanced and simplified variant of LSTM that was initially proposed by (Cho et al., 2014) on statistical machine translation. GRU is inspired by LSTM which controls the information flow inside the unit through update gate $z_{t}$ and reset gate $r_{t}$ without separate memory. Therefore, GRU has the capability of capturing the mapping relationship between timeseries data (Ghazali et al., 2014; Shen et al., 2018) while it also has attractive advantages such as less complexity and efficient computational process. The architecture of GRU, which illustrates the relationship between update and reset gate is presented in Fig4.png.

However, GRU stores and filters information through internal memory capability and integrates the input gate and forget gate into a single update gate with the previous activation $\boldsymbol{h}_{t-1}$ and the candidate state represented by $\tilde{\boldsymbol{h}}_{t}$. There are three major components of GRU are included update gate, reset gate, and candidate state and its equations are as follows:

$$
\begin{gathered}
z_{t}=\varphi\left(V_{x z} x_{t}+U_{h z} h_{t-1}+B_{z}\right) \\
r_{t}=\varphi\left(V_{x r} x_{t}+U_{h r} h_{t-1}+B_{r}\right) \\
\tilde{h}_{t}=\tanh \left(V_{x \tilde{h}} x_{t}+U_{h \tilde{h}}\left(r_{t} * h_{t-1}\right)+B_{\tilde{h}}\right) \\
\left.h_{t}=\left(1-z_{t}\right) * h_{t-1}+z_{t} * \tilde{h}_{t}\right)
\end{gathered}
$$

Where $\boldsymbol{V}_{x Z}, \boldsymbol{V}_{x r}$ and $\boldsymbol{V}_{x \tilde{h}}$ refer to the weight matrix among the input layer and update gate, reset gate, and candidate state while recurrent connection weight matrix is represented by $\boldsymbol{U}_{h z}, \boldsymbol{U}_{h r}$ and 
$304 \boldsymbol{U}_{h \tilde{h}}$ respectively. $\boldsymbol{x}_{t}$ is the time series sample input and hidden output is denoted by $\boldsymbol{h}_{t} \cdot \varphi$ is the 305 sigmoid activation function of update and reset gates, * performs element-wise multiplication 306 operation and $\boldsymbol{B}_{Z}, \boldsymbol{B}_{r}$ and $\boldsymbol{B}_{\tilde{h}}$ are the corresponding biases.

307

308

309

310

311

312

313

314

315

316

317

318

319

320

321

322

323

324

325

326

327

328

329

330

331

332

333

334

335

336

337

\section{PROPOSED METHODOLOGY}

In the research methodology phase, the feature extraction methods have been briefly explained. These approaches are very important to identify the nature of the questions. After that, question classification approaches and classifiers would be examined. Besides, we illustrated modified deep learning architecture which has been utilized in this phase. In our proposed deep learning framework, we demonstrated the process of transforming words into vectors and identifying the questions to relevant classes. After that, for the question classification algorithm, we used Word2vec technique both skip-gram and Continues Bag of Words for classifying questions. In order to extract features, we used one mathematical expression of words. In this mathematical illustration, we allocated every word $x$ to a vector $f(x)$ such that if $x$ and $y$ have syntactic and semantic similarity then $f(y)$ and $f(x)$ will become nearby vectors.

In this study, we performed the multi-fusion $\mathrm{CNN}$ and RNN generated features to conduct the Turkish question classification. In the proposed methodology, we utilized two various modified variants of the recurrent neural network models, such as LSTM and GRU with a combination of CNN.

\section{Modified LSTM}

LSTM is different from standard RNN initially proposed by German researchers Sepp Hochreiter and Juergen in 1997 (Hocheriter \& Schmidhuber; 1997) to learn long-term dependencies. We have explained the traditional LSTM architecture so far as shown in Fig3.png. But there is different LSTM architecture with various equations that help the creation of long-term dependency learning. All LSTMs are not the similar as the traditional ones. One popular variation of LSTM design that includes "peephole connections mechanism" is employed in our methodology (Gers, Schraudolph \& Schmidhuber, 2002). In this section, we used a modified LSTM, which permits the gate layers to expression at the cell state and inserted the "peephole connection mechanism" which directly controls the gates defined as follows:

$$
i_{t}=\varphi\left(W_{x i} \times\left[C_{t-1}, h_{t-1}, x_{t}\right]+b_{i}\right)
$$

$$
f_{t}=\varphi\left(W_{x f} \times\left[C_{t-1}, h_{t-1}, x_{t}\right]+b_{f}\right)
$$

$$
\bar{C}_{t}=\tanh \left(W_{x \bar{c}} *\left[h_{t-1}, x_{t}\right]+b_{\bar{c}}\right)
$$

$$
\left.C_{t}=f_{t} * C_{t-1}+i_{t} * \bar{C}_{t}\right)
$$


338 In these equations, where $\mathrm{x}_{\mathrm{t}}$ is the input transfer matrix of $\mathrm{W}, \mathrm{C}_{\mathrm{t}-1}$ is the memory cell, 339 component-wise multiplication is presented as $\mathrm{x}$, while the hidden state vector $\mathrm{h}_{\mathrm{t}-1}$ and $\varphi$ shows 340 the sigmoid function.

341 The output gate $o_{t}$ control the present hidden state value $h_{t}$, which uses memory cell content

342 for the nonlinearity system result:

$$
o_{t}=\varphi\left(W_{x o} \times\left[C_{t}, h_{t-1}, x_{t}\right]+b_{o}\right)
$$

$$
h_{t}=o_{t} * \tanh \left(C_{t}\right)
$$

345

346

347

348

349

350

351

352

353

354

355

356

357

358

359

360

361

362

363

364

365

366

367

368

According to the following steps, the current stage of the hidden state $h_{t}$ is used for the acquisition of $h_{t+1}$. In other words, long short-term memory processes the word series recursively by computing their internal hidden state $h_{t}$ at each time step. The hidden activation of the final time step can be considered the linguistic description of the complete sequence and fed into the classification layer as input.

\section{Modified GRU}

Initially, GRU was proposed by Cho et al. in 2014 to extract the dependencies of the very recurrent unit at various time steps. GRU is a gating mechanism in RNN and is similar to LSTM with a forget gate. We have explained the traditional GRU architecture so far as shown in Fig4.png. But there is different GRU architecture with various equations that support the development of capturing long-term dependencies.

The mathematical expression of GRU is defined as follows:

$$
\begin{aligned}
& z_{t}=\varphi\left(V_{x z} x_{t}+U_{h z} h_{t-1}+B_{z}\right) \\
& r_{t}=\varphi\left(V_{x r} x_{t}+U_{h r} h_{t-1}+B_{r}\right) \\
& h_{t}=\left(1-z_{t}\right) \odot h_{t-1}+z_{t} \\
& \odot \tanh \left(V_{x_{t}}+U\left(r_{t} \odot h_{t-1}\right)+B_{h}\right)
\end{aligned}
$$

Where $h_{t}$ and $x_{t}$ are the output and input vector at time $t . z_{t}$ and $r_{t}$ are the update and reset gate vector. $\varphi$ and $\odot$ are the sigmoid activation function and element-wise multiplication operation while $B$ are the corresponding bias.

\section{CNN-LSTM/GRU Models}

In this section, we proposed a deep learning hybrid architecture which includes the following parts: word embedding with the Word2vec methods, convolutional neural network, and recurrent 
369

370

371

372

373

374

375

376

377

378

379

380

381

382

383

384

385

386

387

388

389

390

391

392

393

394

395

396

397

398

399

400

401

402

403

404

405

406

neural network with its variants, such as LSTM and GRU, while fully connected layer employed as a softmax output. The word embedding method is applied to convert input text into numerical word vectors to translate into CNN and RNN models. In this way, several convolution kernels of various dimensions were used to capture more helpful features in question classification. With this technique, CNN preserves the temporal data and generates a single value using the maxpooling layer. Similarly, the RNN layer is utilized to obtain the temporal features at the input level and captures long-term dependencies. In word embedding, every question was illustrated as a word embedding matrix to create a classifier using a CNN. Provided a question consisting $n$ words $v_{1}, v_{2}, v_{3}, \ldots v_{n}$ each word with its pre-trained $d$-dimension word embedding matrix is swapped, and stacked row-wise to produce occurrence matrices $V_{i} \in R^{n \times d}$. Moreover, in this study, we trained the hyperparameters using $R e L U$ in the deep learning approaches. Specifically, we selected $R e L U$ because ReLU ("Rectified Linear Unit") ReLU has been widely used in advanced deep learning architecture like CNN, RNN. Fig5.png, shown the essential architecture of the proposed approaches which is adapted from (Yang, Macdonald \& Ounis, 2018; Alayba et al., 2018), and it summarizes the combination of three various deep learning approaches such as CNN, LSTM, and GRU. A quick overview of each layer will be described in more detail.

\section{Input Layer}

The input layer is selected as an initial point of the networks. Let describe the total number of unique words with $w_{1}, w_{2}, w_{3}, \ldots, w_{n}$ in the dictionary $D=d_{1}, d_{2}, d_{3}, \ldots d_{m}$. To identify the questions, the Word2vec model is used to translate any word in the query into a particular vector with one of the fixed sizes of $100,200,300$ or 400 . Every question $j$ is presented with a twodimensional $n \times k$ matrix $c_{j}=\left[v_{1}, v_{2}, \ldots, v_{n}\right]$, the description of $k$ refers to the dimensionality of the $v i$ embedding and a large number of words is denoted by $n$. To provide the same length for all questions the input layer transmits data samples as a sequence of unique indices of similar dimensions.

\section{CNN LAYERS}

The convolution layer is the most useful and basic layer of CNNs that perform the convolution process in the form of row representation through word vectors obtained from the embedding layer. CNN layers contain a set of learnable filters or kernels which map to produce twodimensional activation. Let's considered the $h$ words at time $t$ with weight matrices $\mathrm{w}$ of dimension $w \in R^{h * m}$ to perform the following convolutional computation:

$$
c_{i}=\mathrm{f}\left(X_{i+h-1} * \mathrm{w}+\mathrm{bi}\right)
$$

Where $f$ refers to the non-linear Relu activation function and the feature map generated to represented by $c_{i} \in R_{n-h+1}$ with $h$ words every time frequently, while bias term denoted by $b_{i}$. 
407

408

409

410

411

412

413

414

415

416

417

418

419

420

421

422

423

424

425

426

427

428

429

430

431

432

433

434

435

436

437

438

439

440

441

442

443

444

After that, the max-pooling layer performs to receive created features from convolution which change the features map into its maximum activation value, as follow:

$$
P_{i}=\max C_{i}
$$

where, $P i \varepsilon R^{n-h+1 / 2}$ refers to the new feature map in order to obtain the various levels of features from the convolutional layer, we selected three convolutional layers with filter windows of different sizes 3, 4, and 5. After performing the max-pooling operations, we combined the features from various levels of convolution layers to achieve the final multilevel feature combination output as presented in Fig5.png.

\section{Feature mapping of CNNs layer with RNNs layer}

Each input has a vector series, which scans it with a fixed filter distance. In this technique, the filter sizes of 3, 4, and 5 are used to carry the features of words. The CNNs layers efficiently reduce the input features vector, and give the better-compressed presentation through the maxpooling layer as compared to original raw features and the output generated by CNN's layers are further processed as inputs to the RNNs layers passing through the gating mechanism for learning the high informative features. Furthermore, in order to classify questions every layer processes different features in a question with the Relu activation function in the feature map.

\section{RNN Layers}

RNN layers give exhibit temporal dynamic behavior (Choi et al., 2017) which processes the sequential data within the network. The recurrent layer has the capability to capture the longterm dependencies; therefore we feed original word embedding as an input to the RNN layer instead of those features generated by CNN. The purpose of selecting the RNN layers approach is to take the sequence data through utilizing the previous information. In these RNN layers, the final output of the layers has the equivalent number of units.

Due to sequence data, the RNN layers can learn temporal features from it. After this, we performed a different combination of $\mathrm{CNN}$ and RNN acquired features to carry out question classification. Based on this technique, sequential features should be perfectly maintained and a sequence created by max-pool layer instead of a single value. According to the following process, the data are fed into an RNN layer with many to one mechanism and a fully connected layer with softmax output.

\section{EXPERIMENTAL DESIGN \& DATASET}

We conducted an experimental design to evaluate the effectiveness of five different deep learning approaches on the Turkish question database. Therefore, this section briefly describes the Turkish question database and experimental settings. The overall architecture of this research is presented in Fig6.png. 


\section{Question database description}

447 We evaluate the performance of proposed deep learning approaches on the Turkish dataset for 448 question classification. There is an absence of a Turkish question database as compared to the 449 English database. In this research, we used a dataset of Turkish questions that is adapted from an

450

451

452

453

454

455

456

457

458

459

460

461

462

463

464

465

466

467

468

469

470

471

472

473

474

475

476

477

478

479

480

481

482

English Question Dataset that has been used by Li and Roth's in 2002.

They referred to two-layered classification, which is extensively applied for question categorization. This dataset includes six offensive classes and fifty fine-grained classes that are reported as 'offensive fine' including "LOCATION: city'. This dataset is divided into two parts are training and testing. We experimented to use 5400 questions for training data and the remaining 600 questions for testing data. The distribution of this dataset (Le, Phan \& Nguyen, 2015) categorized into main classes and sub-classes are reported in Table2.doc. In our experiments, we reconstruct the Turkish dataset from the English dataset.

\section{Experimental setting and Hyperparameters}

Deep learning based approaches have the ability to acquire complex relationships among inputs and outputs (Srivastava et al., 2014). In our experiment, we applied Adam optimizer to set their default optimal parameters setting with a learning rate of 0.005 and decay factor is 0.9 . For the CNN layers, we applied three channels where each one uses a two-dimensional convolutional layer with kernel window size 3, 4, and 5. We used the rectified linear unit (ReLU) activation function for each convolutional layer. For each iteration of the training procedure, we fix the batch size to 32. For a fair comparative analysis, a few preprocessing steps are performed to improve the quality of the dataset. However, during the training process, many connections are involved as a result of sampling noise; while it did not exist in the test data. This problem may conduct to overfitting and minimize the prediction ability of the network (Srivastava et al., 2014). For this issue, we applied the dropout method to reduce the overfitting with the dropout probability of 0.2 for recurrent layers and 0.5 after the convolution layer. Moreover, for training the proposed models, we used Cross Entropy with $\mathrm{L}_{2}$ regularization as minimizing the loss function, which referred to as follows:

$$
J(w, b)=-\frac{1}{2} \sum_{i=1}^{m}\left[y_{i} \log \hat{y}_{i}+\left(1-y_{i}\right) \log \left(1-\hat{y}_{i}\right)\right]+\frac{\lambda}{2 m} \sum_{l=1}^{m}\|w\|_{F}^{2}
$$

Where $y_{i}$ is refer ground truth; and classification probability for each class represented by $\hat{y}_{\mathrm{i}}$. We set $\mathrm{w}=0.001$, of Frobenius norm value by compressing $\mathrm{L}_{2}$, which is the coefficient for $\mathrm{L}_{2}$. During the training process, the result presents the $\mathrm{L}_{2}$ regularization and dropout method can perform better to avoid overfitting. Table3.doc, provides the optimal values of hyperparameters, which have been applied for the training of the proposed framework.

Peer] Comput. Sci. reviewing PDF | (CS-2020:10:54597:2:0:NEW 27 Apr 2021) 


\section{Word2vec models}

484 The Word2vec comprehends and vectorizes the meaning of a word in a document established on 485 the hypothesis that words with comparable meaning in a provided context display near distances 486 (Sahlgren, 2008). It is an open-source platform offered by Google in 2013 under the Appache 487 License 2.0. Fig7.png, demonstrates the model architectures of CBOW and Skip-gram learning 488 procedures of Word2vec was referred to by (Nelakantan et al., 2015; Yang et al., 2013). Input, 489 Projection and output layers are present in all the learning algorithm, while their output processes 490 are different. $W_{n}=\left\{W_{(t+2)}, W_{(t+1)}, \ldots, W_{(t+1)}, W_{(t+2)}\right\}$ is received as arguments for the input 491 layer, where $W_{n}$ shows words. The projection layer is a multidimensional vector array that stores 492 the sum of various vectors. The output layer matches the layer which outputs the results of the 493 vectors from the projection layer. It is a shallow of two-layer neural networks that are educated 494 to perform word embedding method. Specially, CBOW is similar to the feedforward Neural

495

496

497

498

499

500

501

502

503

504

505

506

507

508

509

510

511

512

513

514

515

516

517

518

519 Network Language Model (NNLM) (Armeni, Willems \& Frank, 2017) and predicts the output word from other near word vectors. The algorithm of the Word2vec model extracts features from a provided text corpus without any intervention from a human expert. Most essentially, if the text size is too small or only a separate word, it performs quite well. By providing a big corpus, it generates word vectors from a large number of texts and makes it appropriate by comparing the contextual data the input words performed similarly as shown in (https://github.com/akoksal/turkish-word2Vec). In the Word2vec space, every special word in the text is allocated to a connected vector (https://israelg99.github.io/2017-03-23-Word2VecExplained/). The meaning of words is one of the most significant intents in deep learning that are entirely accomplished with employing the Word2vec for classifying major entities (Mikolov et al., 2013). For learning word embeddings from raw data, it is a computationally well-ordered predictive framework. There are two different techniques of Word2vec, as follows.

\section{Skip gram}

\section{CBOW (Continuous Bag of Words)}

Algorithmically, these are two approaches near to each other. By computationally, Continuous Bag of Words (CBOW) is a continuously distributed word representation approach, which classifies the core words (target) based on the neighboring words. The fundamental principle of CBOW includes identifying when a given word comes from neighboring words analysis. The CBOW architecture shows the advantage that the information in the dataset is organized uniformly. Furthermore, the CBOW method derives the dictionary $\|\mathrm{V}\| \in \mathrm{R}^{\mathrm{m}}$ by mapping the words $\left(c_{1}, c_{2}, \ldots, c_{t}\right)$ in the corpus to the projection layer. Then, in the projection layer the corpus word $c_{t}$ is mapped to the unique position $w_{t}$ and refers to the context size by $k$. It is a methodological result that the corpus terms are read sequentially by Continuous Bag of Words [ $\left.c_{t-k}, c_{t-k+1}, \ldots, c_{t+k}\right]$ and achieves the corresponding word position $\left[w_{t-k}, w_{t-k+1}, \ldots, c_{t+k}\right]$ in the projection layer by a hash table. Finally, it performs the following operations on the 
520 Context $\left(w_{t}\right)$ of $w_{t}$, where $V_{t}$ is the context accumulated sum of $w_{t}$ mentioned in equation (14).

521 As one observation, CBOW processes entire contexts sequentially.

522

523

$$
V_{t}=\sum_{t-N}^{t+N} \operatorname{Context}\left(w_{t}\right)
$$

524

525

On the other hand, the skip-gram algorithm is very similar to CBOW as presented in Fig7.png.

526

527

528

529

530

531

532

533

534

535

536

537

538 539

540

541

542

543

544

545

546

547

548

549

550

551

552

553

554

555 The major difference between them is which swaps the output and input, and use the reverse functionality to each other. Skip-gram is typically used to predict all background terms in a single input word for a given target word. The projection layer of the Skip-gram predicts next words around the inserted into the input layer. The Skip-gram architecture shows the advantage of vectorizing when new words appear. Generally, the training purpose of the skip-gram method usually discovers word vectors that help to identify near words in the relevant contexts (Nooralahzadeh, Ovrelid \& Lonning, 2018). The skip-gram model shows a mechanism to predict a one-word vector from other words ("opposite of Continuous Bag of Words"). We summarize it and describe our notations for the execution skip-gram approach. Suppose words of corpus and their contexts: $D=(w, c)=\left(w_{1}, c_{1}\right),\left(w_{2}, c_{2}\right) \ldots\left(w_{n}, c_{n}\right) ; w_{i} \in V_{W}, c_{i} \in V_{C}$, which are one-hot vectors, where $V_{W}$ is present the word vocabulary and $V_{C}$ is present the context vocabulary with sizes of $S_{W}$ and $S_{C}$ respectively. The learning procedure of skip-gram (SG) attempts to train the contextual distribution for separate word by optimizing the prospect function as follows:

$$
S G(\boldsymbol{c} / \boldsymbol{w} ; E, F)=\prod_{i=1}^{n} S G\left(c_{i} / w_{i} ; E, F\right)=\prod_{i=1}^{n} \frac{\exp \left(w_{i}^{T} E F\right) c_{i}}{\sum_{\bar{c} \in C} \exp \left(w_{i}^{T} E F\right) \bar{c}}
$$

Where $E$ and $F$ are the parameter matrix of the shapes $\left(S_{W} \times d\right)$ and $\left(d \times S_{C}\right)$ respectively. $d$ is the dimensionality of the embedding vector space. Contrary, $P\left(x_{i o}=1 \mid w_{i}, c_{i} ; E, F\right)$ presents specific contexts that appear close to a word or not. While, in this study, we refer $P(D ; \theta)$ for the skip-gram training demonstrated by $P_{S G}(\boldsymbol{c} \mid \boldsymbol{w} ; E, F)$. The skip-gram algorithm works more effectively on a big corpus, every context-center pair is handled as a new consideration. The architecture of skip-gram (sg) and Continuous Bag of Words (CBOW) are shown in Fig7.png.

\section{Training Word2vec embedding model}

As part of this research, we have the selected most widely applied deep learning architecture with the Word2vec model that focuses on question classification. For word embedding, we implemented various parameters in order to train Word2vec of both Skip gram and CBOW models on Wikipedia corpora. We explored and trained our Word2vec models on a Turkish Wikipedia dataset for question classification. As the largest encyclopedia in which documents are well organized by topics on the Internet, we preferred it as the dataset. Therefore, Wikipedia 
556

557

558

559

560

561

562

563

564

565

566

567

568

569

570

571

572

573

574

575

576

577

578

579

580

581

582

583

584

585

586

587

588

589

590

591

592

corpora are well suitable for the analysis of the Word2vec approach. However, in our study, we eliminate unnecessary words from the question classification corpus with less than 5 words during the training on Wikipedia corpus. Because these words have minor quantities of data which are usually helpless for training the Word2vec model (e.g., some have stop words, streaming, and emotions). Moreover, we conducted an experiment by using Wikipedia corpus, to build our skip-gram and CBOW model with various vectors lengths 100, 200, 300, and 400. In addition, to avoid the overfitting issues, we used the dropout technique (Hinton, 2014), with a dropout rate of 0.5 for convolutional layers while 0.2 for recurrent layers.

For all the experiments, to train our Word2vec model, we used Gensim (Liu et al., 2018) to generate a set of word embeddings by setting the context window size $W$, the dimensionality $D$, the complete number of negative samples is presented by $n s$ and the skip-gram is shown by $s g$. The value of the predefined parameter for the context window size is selected as $\mathrm{W}=\{5\}$. Similarly, for this window size, to explore both of the high and low dimensions, we applied four various dimensionality sizes $D=\{100,200,300,400\}$ for the word2vec vectors. Therefore, we have chosen the default parameters for the training of deep learning approaches with the word2vec model. We fixed 5 as the negative sampling, batch words to 10,000 minimum counts of words to 5 and iteration to 5 . In addition, we analyzed the effect of the dimensional vectors on the Turkish question dataset, while it includes near about one million Turkish articles and has been explored on Turkish Wikipedia. After removing the noisy words that are frequently less than 5, more than 200 thousand Turkish words are collected in the database. The parameters of deep learning approaches employing the Word2vec models are presented in Table3.doc.

Additionally, in this study accuracy was selected as an evaluation metric. It evaluates the correctness of the model and is calculated as the ratio of correctly classified instances (TP) divided by the total numbers of instances $(\mathrm{TP}+\mathrm{FP})$ on the entire dataset. The formula of accuracy in question classification defined as follows:

$$
\text { Accuracy }=\frac{T P_{(n)}^{(m)}+T N_{(n)}^{(m)}}{T P_{(n)}^{(m)}+F P_{(n)}^{(m)}+F N_{(n)}^{(m)}+T N_{(n)}^{(m)}}
$$

Where, TP and $T N$ present True Positive, True Negative respectively, which indicate the correct classification for the relevant class while $F P$ and $F N$ refer to the False Positive, and False Negative which determine the false classification for the relevant class. Particularly, in word embedding, we utilized the Word2vec model both of skip-gram and CBOW as feature extraction models. Furthermore, in terms of 10-cross validation accuracy, we also compared the results.

\section{EXPERIMENTAL RESULTS}

In this part, we illustrate the performance of different deep learning algorithms using Word2vec embedding vectors of both $\mathrm{CBOW}$ and the skip-gram methods on the question dataset. The semantic and syntactic connections between words can be efficiently captured by these 
593 techniques. In this way, initially, the Word2vec model calculates word vectors in the vocabulary 594 words. Similarly, the word 2 vec model initializes and selects random vectors from word vectors. 595 Then, these algorithms attempt to increase the cosine similarity between all terms and their 596 contexts, which is described based on the method. Consequently, these algorithms will be 597 capable to allowing word vectors from a large amount of text of the Wikipedia corpus, while 598 their nearness is associated with their corresponding words.

599 These deep learning techniques applied for questions classification is based upon the 600 Word2vec models of skip-gram and CBOW with random vector approaches. To the best of our 601 knowledge, this research concentrates on agglutinative language even this is the first time studied 602 in an agglutinative language in detail for question classification. As a performance analysis, we

603

604

605

606

607

608

609

610

611

612

613

614

615

616

617

618

619

620

621

622

623

624

625

626

627

628

629

630

631

632 experimented based on the word $2 \mathrm{vec}$ models and achieved satisfactory results in a term of accuracy. Table4.doc to Table8.doc, compare the results of deep learning models with both word $2 \mathrm{vec}$ word embedding techniques as a function of training volume based on the fixed epoch in question classification.

\section{DISCUSSION}

This section analyzes the competitiveness and effectiveness of our proposed results with various deep learning approaches with both word2vec word embedding techniques by taking into consideration an accuracy. Table4.doc to Table8.doc, show the accuracy comparison of deep learning models based on the various number of feature vectors with Word2Vec methods. The previous research on question classification focuses on various tasks of occurrence for relevant class categorization or named entities (Derici et al., 2015). However, they integrated a rule-based method employing an HMM-based sequential categorization method (Donmez \& Adali, 2017). And their answers could not generalise to question classification tasks in an agglutinative language. Furthermore, some alternate studies have been performed on similar language work for questions classification et (Razzaghnoori al., 2018); (https://github.com/thtrieu/qclass dl/blob/master/ProjectDescription.pdf,https://github.co $\mathrm{m} /$ thtrieu/qclass_dl/blob/master/Project Presentation.pdf) has not examined the influence of the Word2vec models on both variants skip-gram and CBOW. Particularly, to evaluate the performance of question classification, they applied some parameters such as feature vector size, window size. In this study, the experimental results demonstrate the factors that those mentioned-above can certainly affect the performance of the question classification system.

In our research, generally, we examined four different deep learning models including CNN, GRU, LSTM, CNN-LSTM, and CNN-GRU based on Word2vec models of both CBOW and skip gram. However, by comparison, the CNN, CNN-LSTM and CNN-GRU models are capable to achieved significantly superior results in the term of accuracy when using skip-gram model on Turkish questions classification dataset as compared to CBOW model (Tables 4.doc, 7.doc, 8.doc). On the other hand, CNN, CNN-LSTM, and CNN-GRU, commonly perform better than LSTM and GRU architectures by using CBOW model. In most of the cases, the CNN-LSTM and CNN-GRU approach achieved better results based on skip-gram than CBOW. Moreover, we 
633 have observed an excellent result in CNN approach, an accuracy of $93.7 \%$, based on the skip634 gram with 300 feature vectors. Furthermore, we experienced that when utilizing the correct form 635 of a dataset can probably incorporate more vocabulary for the question classification database. 636 For this reason, the correlation between corpus and the classification dataset provides better 637 question-level representation. Finally, on the same dataset, we compared the performance of our 638 proposed approaches with a similar 639 ((https://github.com/thtrieeu/qclass_dl/blob/master/ProjectDescription.pdf,), in which the 640 researchers have used the LSTM approach to obtain 94.4\% accuracy in English language; we 641 noticed that our achieved results were low compared to this study carried out in English. The

642 main cause behind of this is the Turkish language construction as we already described above in 643 the introduction section. As a result, there is a lack of efficient Turkish Language lemmatization 644 tools compared to English language.

645

646 CONCLUSIONS

647 Question classification is an important area in natural language processing (NLP). Recently, 648 there are several deep learning models have been used to solve these issues and have shown 649 remarkable results in NLP. In this study, we applied four different deep learning approaches to 650 the question dataset using Word2vec embedding vectors both skip-gram and CBOW models. 651 We noticed that the use of Word2vec models can efficiently learn the text semantic and syntactic 652 connections between words significantly improved the performance of the classification models. 653 In this research, initially, the Word2vec methods compute the word vectors from vocabulary 654 words and initialized with random vectors. Therefore, by applying a huge amount of text for 655 these algorithms generated by Wikipedia corpus, they will be capable to allow word vectors in 656 the vector space such that their closeness is corresponding to their associated words. By 657 comparative analysis, all deep learning models have revealed superior performance with 658 word2vec models in the tasks of question classification. However, in some of the cases, we 659 observed that the skip gram model performed well as compared to CBOW model.

In our future direction, for further improve the performance we recommend here in one sentence that may motivate us to explore for future work. To improve the accuracy of hybrid feature extraction techniques can be applied for more than one-word embedding methods together for a question classification system. Hopefully, the system will be capable of acquiring the advantages for all embedding techniques when using this hybrid method.

665

\section{ACKNOWLEDGEMENTS}

667 The authors would like to thank the Ministry of Higher Education Malaysia (MOHE) and 668 Universiti Tun Hussein Onn Malaysia for funding this research activity under the Fundamental 669 Research Grant Scheme (FRGS/1/2017/ICT02/UTHM/02/5), vote no. 1641.

670

\section{REFERENCES}


672 Wang Z, \& Qu, Z. 2017. Research on Web text classification algorithm based on improved CNN and SVM. In

6732017 IEEE 17th International Conference on Communication Technology (ICCT): pp. 1958-1961. IEEE.

674 Sharif W, Samsudin N. A, Deris M. M, Aamir M. 2017. Improved relative discriminative criterion feature ranking 675 technique for text classification. International Journal of Artificial Intelligence 15(2): 61-78

676 Le-Hong P, Phan XH, Nguyen TD. 2015. Using dependency analysis to improve question classification. In:

677 Knowledge and systems engineering. Advances in intelligent systems and computing vol (326):653-665

678 Mohd M, \& Hashmy R. 2018. Question classification using a knowledge-based semantic kernel. In Soft 679 Computing: Theories and Applications (pp. 599-606), Singapore.

680 Armeni K, Willems R. M, Frank S. L. 2017. Probabilistic language models in cognitive neuroscience. Promises 681 and pitfalls. Neuroscience \& Biobehavioral Reviews (83): 579-588.

682 Mikolov T, Sutskever I, Chen K, Corrado GS, Dean J. 2013. Distributed representations of words and phrases 683 and their compositionality. In Advances in neural information processing systems 4(11): 3111-3119

684 Liu M, Lang B, Gu Z, Zeeshan A. 2017. Measuring similarity of academic articles with semantic profile and joint 685 word embedding. Tsinghua Science and Technology 22(6): 619-632.

686 http://cogcomp.cs.illinois.edu/Data/QA/QC/

687 Ehrentraut C, Ekholm M, Tanushi H, Tiedemann J, Dalianis H. 2018. Detecting hospital-acquired infections: a 688 document classification approach using support vector machines and gradient tree boosting. Health informatics 689 journal 24(1): 24-42.

690 Madabushi HT, Lee M, Barnden J. 2018. Integrating question classification and deep learning for improved 691 answer selection. In Proceedings of the 27th International Conference on Computational Linguistics (pp. 3283692 3294).

693 Athira PM, Sreeja M, Reghuraj PC. 2013. Architecture of an ontology-based domain-specific natural language 694 question answering system. International Journal of Web SemantTechnology 4(4):31

695 Razzaghnoori M, Sajedi H, Jazani IK. 2018. Question classification in Persian using word vectors and 696 frequencies. Cognitive Systems Research 9(47):16-27

697 Ozturkmenoglu O, Alpkocak A. 2012. Comparison of different lemmatization approaches for information retrieval 698 on Turkish text collection. In: 2012 International symposium on innovations in intelligent systems and applications. 699 IEEE.

700 Hao T, Xie W, Xu F. 2015. A WordNet expansion-based approach for question targets identification and 701 classification. In Chinese computational linguistics and natural language processing based on naturally annotated 702 Big Data. Springer, Cham, pp 333-344.

703 Oflazer K (2014). Turkish and its challenges for language processing. Lang Resour Eval 48(4):639-653

704 Le-Hong P, Phan XH, Nguyen TD. 2015. Using dependency analysis to improve question classification. In 705 Knowledge and Systems Engineering (pp. 653-665). Springer, Cham

706 Galitsky B (2017). Matching parse thickets for open domain question answering. Data \& Knowledge Engineering, 707 (107), 24-50.

708 Sarrouti M, and El Alaoui S.O. 2017. A machine learning-based method for question type classification in 709 biomedical question answering. Methods of information in medicine, 56(03): 209-216.

710 Ehsan S, Mojgan F. 2014. A hybrid approach for question classification in Persian automatic question answering 711 systems 2014. In 4th international e conference on computer and knowledge engineering (ICCKE). IEEE, pp 279712 284(2014)

713 Sahlgren M. 2008. The distributional hypothesis. Italian Journal of Disability Studies Vol. 20, pp 33-53.

714 Sherkat E, Farhoodi M. 2014. A hybrid approach for question classification in Persian automatic question 715 answering systems. In 2014 4th International Conference on Computer and Knowledge Engineering (ICCKE) (pp. 716 279-284). IEEE. 
717 Huang X, Maier A, Hornegger J, Suykens JA. 2017. Indefinite kernels in least squares support vector machines

718 and principal component analysis. Applied and Computational Harmonic Analysis, 43(1): pp 162-172.

719 Chitra S, Kalpana B. 2013. Hierarchical Directed Acyclic Graph (HDAG) Based Preprocessing Technique for

720 Session Construction. In Advances in Computing and Information Technology (pp. 611-621). Springer, Berlin,

721 Heidelberg.

722 Xu D, Jansen P, Martin J, Xie Z, Yadav V, Madabushi HT, Clark P. 2019. Multi-class hierarchical question

723 classification for multiple choice science exams. arXiv preprint arXiv:1908.05441.

Merchant K. Pande Y. 2018. Nlp based latent semantic analysis for legal text summarization. In 2018 International Conference on Advances in Computing, Communications and Informatics (ICACCI) (pp. 1803-1807). IEEE.

Zulqarnain M, Ghazali R, and Hassim YMM. 2019. A comparative review on deep learning models for text classification. Indonesian Journal of Electrical Engineering and Computer Science. 1(4): 886-996.

Hochreiter; Sepp; \&Schmidhuber, J.; Long Short-Term Memory. Neural Computation, 9(8), 1735-1780 (1997) 730 https://doi.org/10.1162/neco.1997.9.8.1735

731 Gers, F.A, Schraudolph, N.N, \& Schmidhuber, J. 2002. Learning precise timing with LSTM recurrent networks. 732 Journal of machine learning research, 3(Aug), 115-143.

733

734

735

736

737

738

739

740

741

742

743

744

745

746

747

748

749

750

751

752

753

754

755

756

757

758

759

760

Samarawickrama, A. J. P.; \& Fernando, T. G. I. 2018. A recurrent neural network approach in predicting daily stock prices an application to the Sri Lankan stock market. 2017 IEEE International Conference on Industrial and Information Systems, ICIIS 2017 - Proceedings, 2018-Janua, 1-6

Lee, H.; For Modeling Sentences and Documents, Proc. 15th Annu.Conf. North Am. Chapter Assoc. Comput., no. June, pp. 1512-1521 (2015)

Cho, K.; van Merrienboer, B.; Gulcehre, C.; Bahdanau, D.; Bougares, F.; Schwenk, H.; \&Bengio, Y.; Learning Phrase Representations using RNN Encoder-Decoder for Statistical Machine Translation. ArXiv, (September), 13(3) 1-15 (2014)

Ghazali R, Husaini NA, Ismail LH, Herawan T, Hassim YMM. The performance of a Recurrent HONN for temperature time series prediction.ProcIntJtConf Neural Networks.2014;(July):518-24.

Shen G, Tan Q, Zhang H, Zeng P, Xu J. ScienceDirect Deep Learning with Gated Recurrent Unit Networks for Financial Deep Learning with Gated Recurrent Unit Networks for Financial Sequence Predictions Sequence Predictions. Procedia Comput Sci [Internet]. 2018;131:895-903. Available from: https://doi.org/10.1016/j.procs.2018.04.298

Yang X, Macdonald C, Ounis I (2018) Using word embeddings in twitter election classification. InfRetr J 21(23): $183-207$

https://github.com/thtrieu/qclass_dl/blob/master/ProjectDescription.pdf, https://github.com/thtrieu/qclass_dl/blob/master/Project Presentation.pdf

Alayba AM, Palade V, England M, Iqbal R (2018) A combined CNN and LSTM model for Arabic sentiment analysis. In: International cross-domain conference for machine learning and knowledge extraction. Springer, Cham, pp 179-191

Neelakantan, A., Shankar, J., Passos, A., \& McCallum, A. (2015). Efficient non-parametric estimation of multiple embeddings per word in vector space. arXiv preprint arXiv:1504.06654.

Yang L, Pan T, Yang T, Wang S, Tang J, and Cambria E. (2017) "Learning word representations for sentiment analysis." Cognitive Computation 9, no. 6, pp 843-851.

https://github.com/akoksal/turkish-word2Vec

https://israelg99.github.io/2017-03-23-Word2Vec-Explained/

https://medium.com/@mubuyuk51/word2vec-nedir-t\%C3\% BCrk\%C3\%A7e-f0cfab20d3ae

PeerJ Comput. Sci. reviewing PDF | (CS-2020:10:54597:2:0:NEW 27 Apr 2021) 
761 Nooralahzadeh, F., Øvrelid, L., \& Lønning, J. T. (2018). Evaluation of domain-specific word embeddings using 762 knowledge resources. In Proceedings of the eleventh international conference on language resources and evaluation 763 (LREC 2018).

764 Liu S, Bremer PT, Thiagarajan JJ, Srikumar V, Wang B, Livnat Y, Pascucci V (2018) Visual exploration of

Derici C, Celik K, Kutbay E, Aydın Y, Güngo“r T, O“ zgu“r A, Kartal G (2015) Question analysis for a closed domain question answering system. In: International conference on intelligent text processing and computational 769 linguistics. Springer, Cham, pp 468-482.

770 Srivastava N, Hinton GE, Krizhevsky A, Sutskever I, Salakhutdinov R (2014) Dropout: a simple way to prevent 771 neural networks from overfitting. J Mach Learn Res 15(1):1929-1958

772 Donmez I', Adalı E (2017) Turkish question answering application with course-grained semantic matrix 773 representation of sentences. In: Computer science and engineering (UBMK), 2017 international conference on 774 IEEE, pp 6-1

775 Choi.K, G. Fazekas, B. Mark Sandler, and K. Cho (2017) Convolutional recurrent neural networks for music 776 classication," in Proc. ICASSP, New Orleans, LA, USA, pp. 2392-2396.

777 G. Hinton, “Dropout : A Simple Way to Prevent Neural Networks from Overfitting," J. Mach. Learn. Res. 2014, 15, 778 vol. 15, pp. 1929-1958, 2014. 
Figure 1

The general architecture of NLQA system 


\section{Question/Query}

NLP Tools

\section{Question Processing}

\section{Document Processing}

\section{Answer Extraction}


Figure 2

\section{Conventional CNNs architecture for question classification}

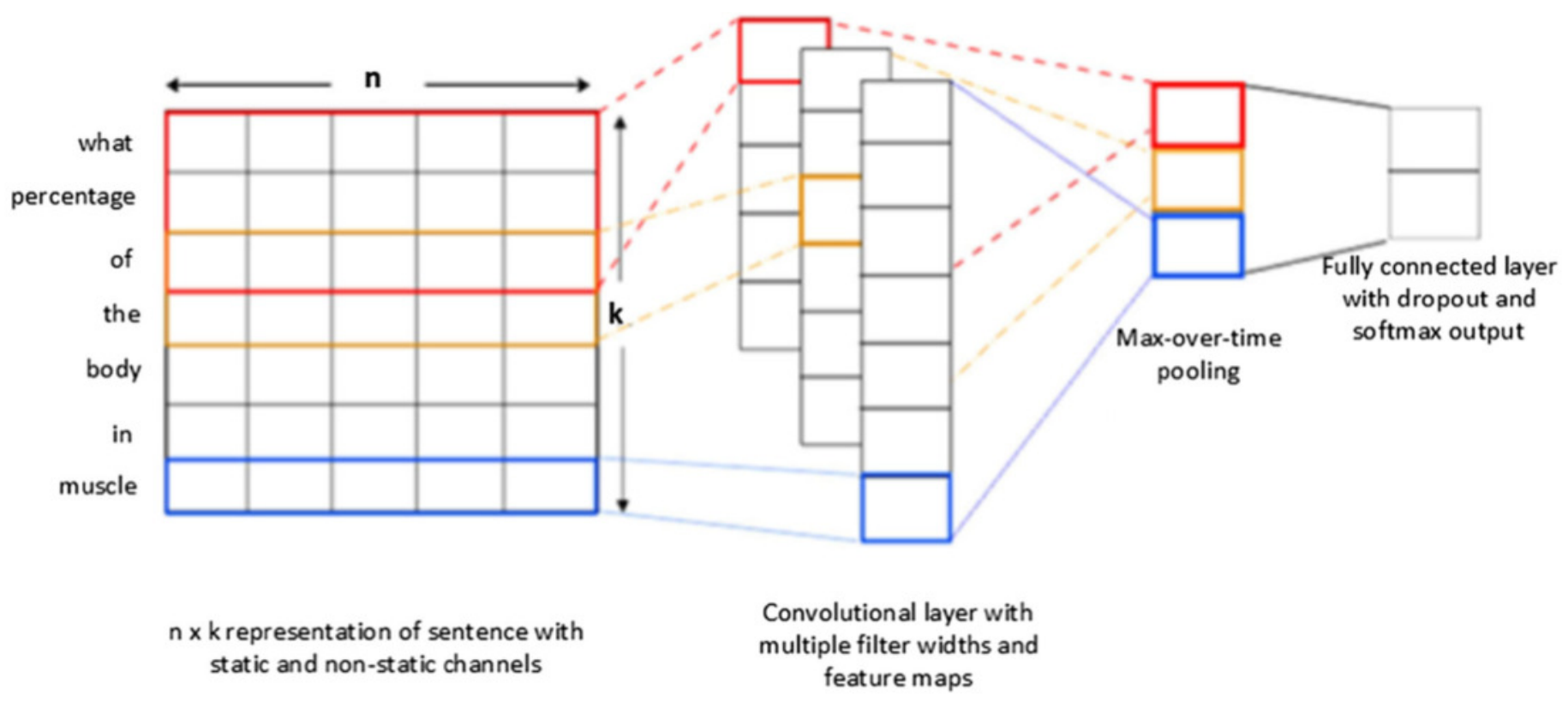


Figure 3

\section{LSTM Structure Diagram}

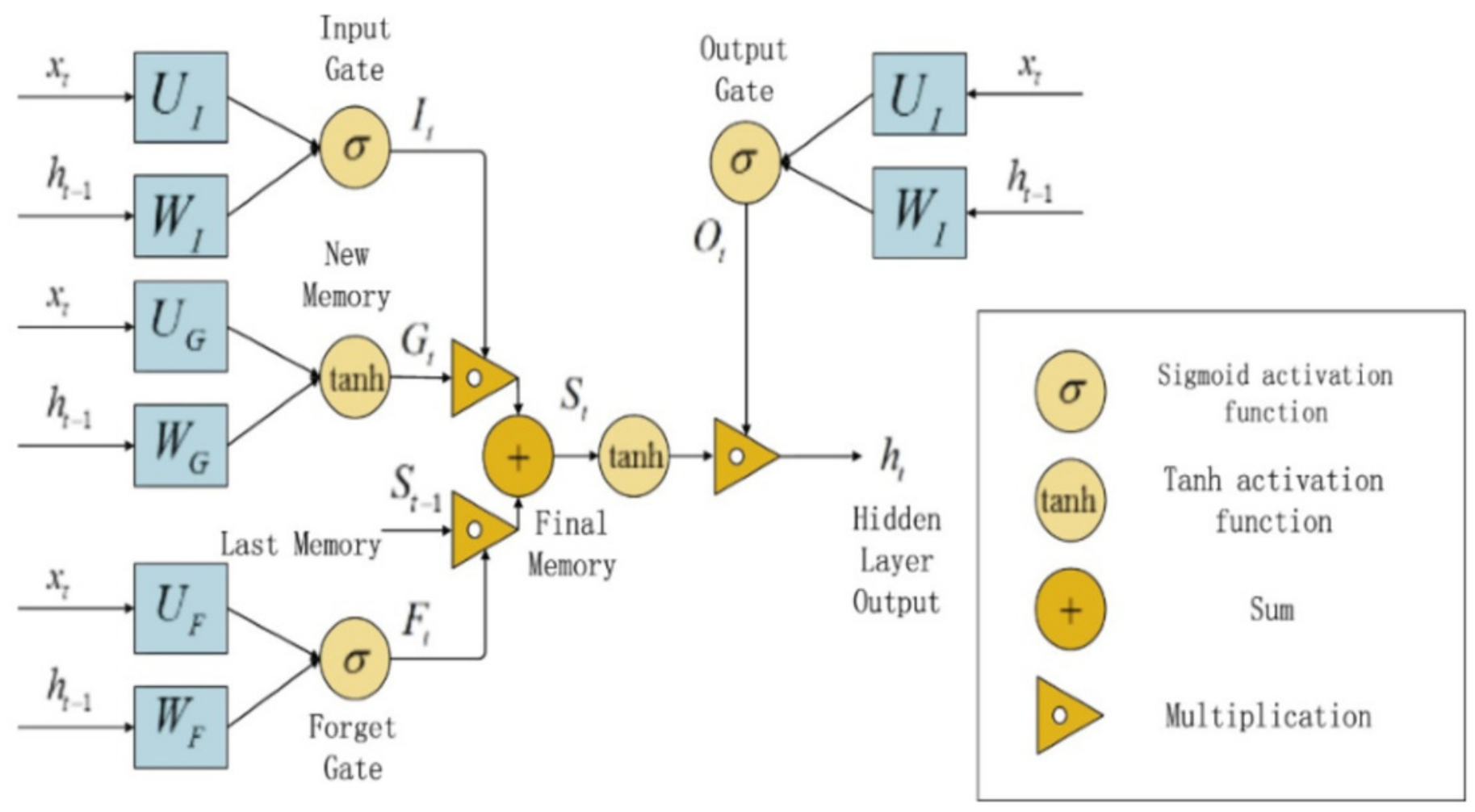


Figure 4

\section{GRU Architecture}

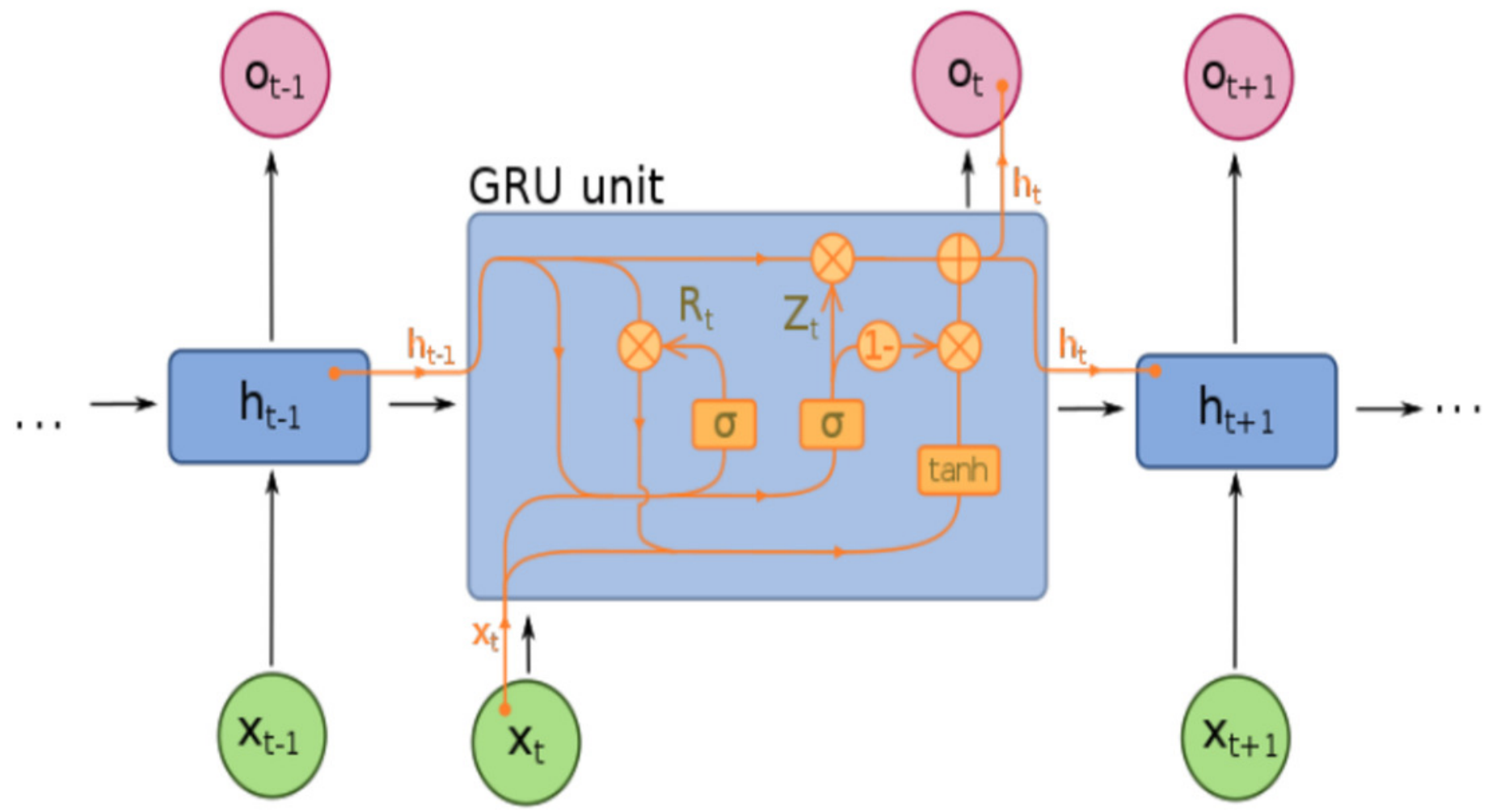


Figure 5

\section{CNN-LSTM/GRU architecture with an example}

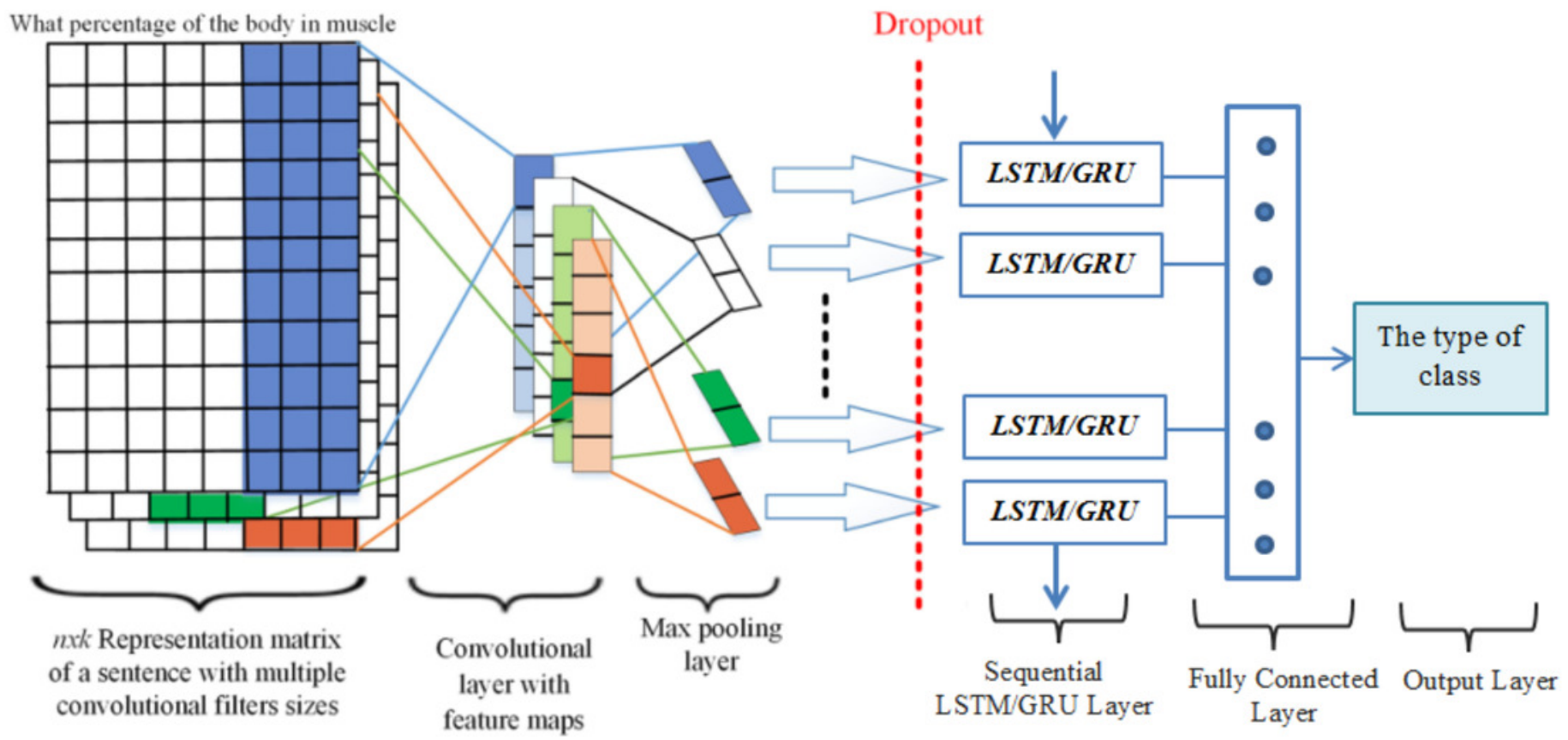


Figure 6

the general architecture of this study

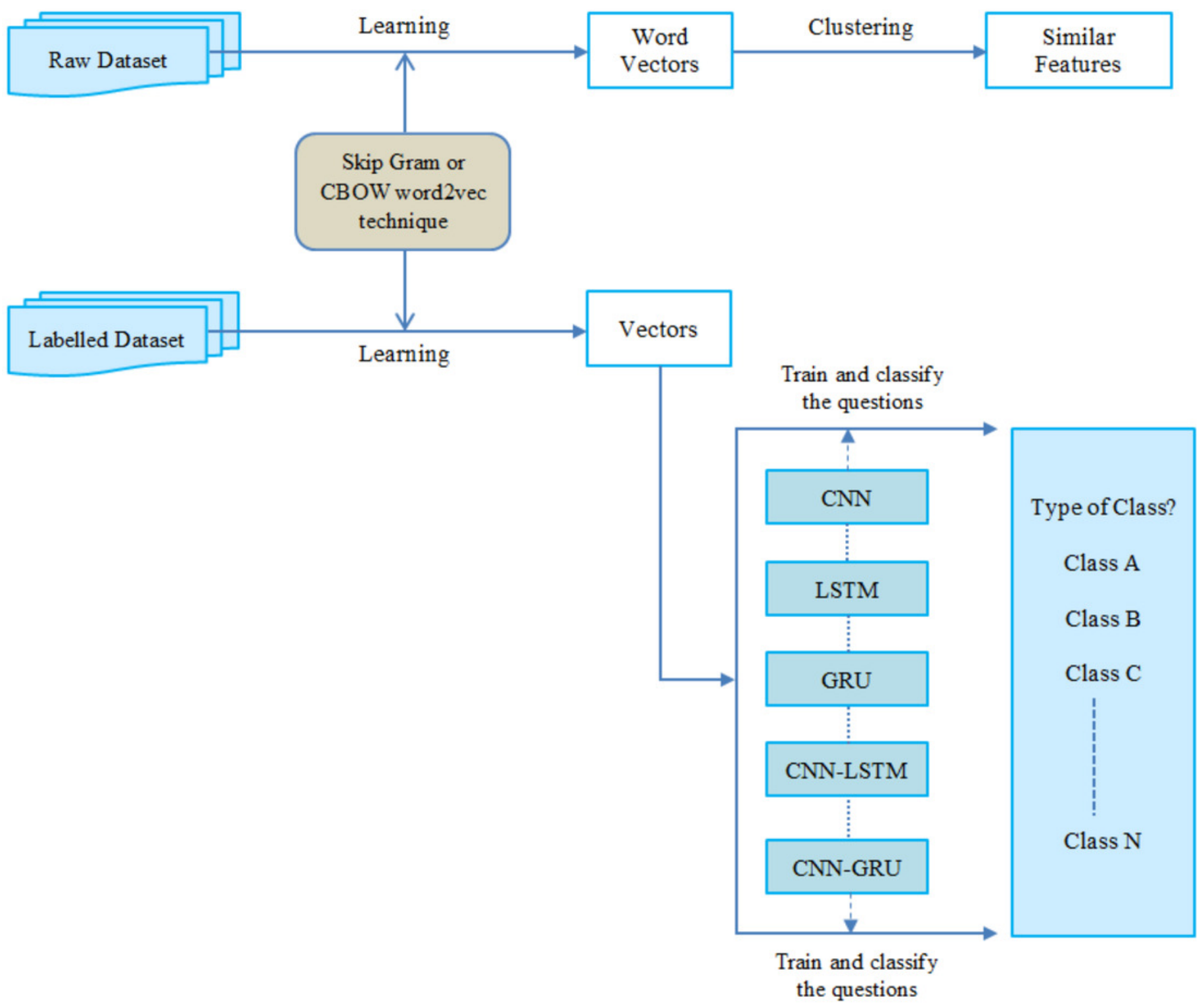


Figure 7

The (A) CBOW and (B) Skip gram model

(A) $\mathrm{CBOW}$, (B) Skip gram

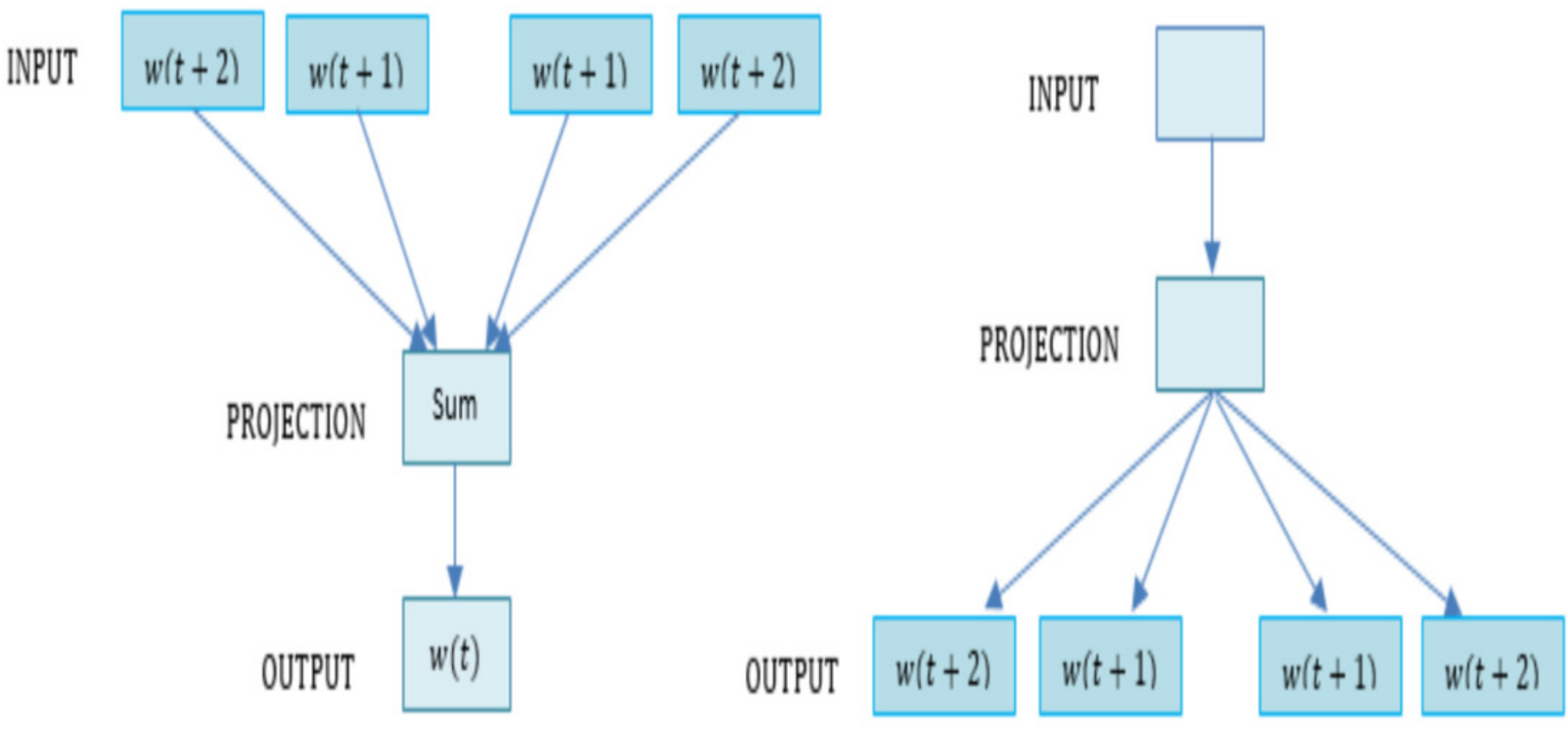

(A) $\mathrm{CBOW}$

(B) Skip-gram 


\section{Table $\mathbf{1}$ (on next page)}

Summary of related researches 


\begin{tabular}{|c|c|c|c|c|c|}
\hline Language & Dataset & Feature Extraction algorithm & $\begin{array}{l}\text { Classification } \\
\text { Approach }\end{array}$ & $\begin{array}{l}\text { Accuracy } \\
(\%)\end{array}$ & References \\
\hline English & $\begin{array}{l}\text { UIUC question } \\
\text { dataset }\end{array}$ & Bag of n-grams & SVM & 87.4 & $\begin{array}{l}\text { (Dell and Wee, } \\
\text { 2003) }\end{array}$ \\
\hline English & $\begin{array}{c}\text { UIUC questions } \\
\text { dataset }\end{array}$ & Bag of n-grams & DT & 84.4 & $\begin{array}{l}\text { (Dell and Wee, } \\
\text { 2003) }\end{array}$ \\
\hline English & $\begin{array}{l}\text { UIUC question } \\
\text { dataset }\end{array}$ & $\begin{array}{c}\text { To produce more complicated features, } \\
\text { such as named entities, } \\
\text { Words, head chunks, chunks, POS tags, } \\
\text { semantically } \\
\text { related words and through these } \\
\text { fundamental features few } \\
\text { operators are utilized }\end{array}$ & SNoW & 91 & (Close, 2002) \\
\hline English & NTCIR-QAC1 & $\begin{array}{l}\text { Extract semantic information over named } \\
\text { entities and words }\end{array}$ & $\begin{array}{l}\text { SVM using } \\
\text { HDAG kernel }\end{array}$ & 88.0 & $\begin{array}{l}\text { (Suzuki et al., } \\
\text { 2003) }\end{array}$ \\
\hline English & $\begin{array}{l}\text { UIUC questions } \\
\text { dataset }\end{array}$ & $\begin{array}{c}\text { WordNet semantic features for n-grams, } \\
\text { word shape, } \\
\text { headword, wh-words, headword }\end{array}$ & SVM and ME & 89 & $\begin{array}{l}\text { (Zhiheng et al., } \\
\text { 2008) }\end{array}$ \\
\hline English & UIUC & $\begin{array}{c}\text { Headwords, word-shapes, associated } \\
\text { words, hypernyms, } \\
\text { Bi-grams, wh-words }\end{array}$ & NB & 93.8 & $\begin{array}{l}\text { (Loni et al., } \\
\text { 2011) }\end{array}$ \\
\hline Persian & UTQD.2016 & Word2vec, CBOW & $\begin{array}{l}\text { LSTM, SVM, } \\
\text { RNN and } \\
\text { MLP }\end{array}$ & 81.77 & $\begin{array}{l}\text { (Razzaghnoori } \\
\text { et al., 2018) }\end{array}$ \\
\hline Persian & $\begin{array}{l}\text { QURANIC } \\
\text { questions }\end{array}$ & $\begin{array}{c}\text { N-gram, POS tags, Lemma, Normalized } \\
\text { word, special word detection and length } \\
\text { of question }\end{array}$ & $\begin{array}{l}\text { SVM with } \\
\text { determined } \\
\text { instruction }\end{array}$ & 80.5 & $\begin{array}{l}\text { (Ehsan \& } \\
\text { Mojgan, 2014) }\end{array}$ \\
\hline
\end{tabular}




\section{Table 2 (on next page)}

Distribution summary of question categories in the UIUC Question dataset 


\begin{tabular}{|c|c|c|c|c|c|}
\hline Category & Training & Testing & Category & Training & Testing \\
\hline DESCRIPTION & 1159 & 96 & HUMAN & 1218 & 68 \\
\hline Reason & 190 & 8 & Other & 216 & 12 \\
\hline Manner & 274 & 2 & Description & 26 & 4 \\
\hline Definition & 422 & 124 & Group & 48 & 8 \\
\hline $\begin{array}{c}\text { ABBREVIATIO } \\
\text { N }\end{array}$ & 84 & 9 & Creative & 208 & 0 \\
\hline Currency & 4 & 4 & Animal & 110 & 15 \\
\hline Religion & 4 & 0 & Individual & 190 & 58 \\
\hline Plant & 14 & 6 & Title & 960 & 1 \\
\hline ENTITY & 1252 & 96 & LOCATION & 836 & 82 \\
\hline Body & 15 & 3 & City & 130 & 20 \\
\hline Letter & 9 & 0 & State & 70 & 10 \\
\hline Instrument & 10 & 2 & Mountain & 24 & 5 \\
\hline Symbol & 11 & 3 & Country & 152 & 4 \\
\hline Lang & 16 & 2 & NUMERIC & 900 & 226 \\
\hline Technique & 34 & 6 & Temp & 8 & 4 \\
\hline Word & 25 & 3 & Date & 221 & 48 \\
\hline Vehicle & 28 & 5 & Weight & 12 & 4 \\
\hline Product & 40 & 5 & Code & 8 & 2 \\
\hline Substance & 40 & 14 & Speed & 10 & 5 \\
\hline Color & 40 & 10 & Period & 30 & 9 \\
\hline Food & 102 & 4 & Size & 16 & 0 \\
\hline Term & 94 & 8 & Distance & 35 & 15 \\
\hline Sport & 62 & 1 & Money & 70 & 4 \\
\hline Event & 58 & 4 & Count & 368 & 9 \\
\hline & & & & & \\
\hline
\end{tabular}




\section{Table 3 (on next page)}

Summary of major parameters utilized in the deep learning approaches with Word2vec models 


\begin{tabular}{|c|c|c|c|}
\hline Parameters & Value & Parameters & Value \\
\hline Dimensionality & $100,200,300,400$ & Dropout & $0.2,0.5$ \\
\hline Sample & 0.001 & Seed & 1 \\
\hline Window size & 10 & min_alpha & 0.0001 \\
\hline Sentences & None & min_count & 5 \\
\hline Batch_words & 10,000 & cbow_mean & 0.025 \\
\hline Max_vocab_size & None & Alpha & 0 \\
\hline SG[0,1] & $\begin{array}{c}1 \text { for skim gram } \\
\text { otherwise CBOW }\end{array}$ & null_word & ReLU \\
\hline HS[0,1] & 0 & Activation function & None \\
\hline Negative & 5 & trim_rule & 1 \\
\hline Hashfxn & 5 & sorted_vocab & \\
\hline
\end{tabular}




\section{Table 4(on next page)}

The results of CNN model using Word2vec 


\begin{tabular}{|c|c|c|c|}
\hline Number of feature vectors & Type of Word2vec model & Accuracy (Test) & $\begin{array}{c}\text { Accuracy (10-cross fold } \\
\text { validation) }\end{array}$ \\
\hline 100 & CBOW & 92.1 & 86.0 \\
\hline 100 & Skip gram & 92.4 & 88.8 \\
\hline 200 & CBOW & 92.2 & 86.3 \\
\hline 200 & Skip gram & 92.1 & 88.9 \\
\hline 300 & CBOW & 91.9 & 90.6 \\
\hline 300 & Skip gram & 93.7 & 87.0 \\
\hline 400 & CBOW & 91.8 & 89.2 \\
\hline 400 & Skip gram & 93.5 & \\
\hline
\end{tabular}




\section{Table 5 (on next page)}

The Results of LSTM using Word2vec model 


\begin{tabular}{|c|c|c|c|}
\hline Number of feature vectors & Type of Word2vec model & Accuracy (Test) & $\begin{array}{c}\text { Accuracy (10-cross fold } \\
\text { validation) }\end{array}$ \\
\hline 100 & CBOW & 90.9 & 86.9 \\
\hline 100 & Skip gram & 91.2 & 87.0 \\
\hline 200 & CBOW & 90.8 & 87.9 \\
\hline 200 & Skip gram & 90.7 & 87.5 \\
\hline 300 & CBOW & 90.4 & 87.1 \\
\hline 300 & Skip gram & 91.3 & 88.7 \\
\hline 400 & CBOW & 91.0 & 88.2 \\
\hline 400 & Skip gram & 90.8 & \\
\hline
\end{tabular}




\section{Table 6 (on next page)}

The Results of GRU using Word2vec model 


\begin{tabular}{|c|c|c|c|}
\hline Number of feature vectors & Type of Word2vec model & Accuracy (Test) & $\begin{array}{c}\text { Accuracy (10-cross fold } \\
\text { validation) }\end{array}$ \\
\hline 100 & CBOW & 91.5 & 86.5 \\
\hline 100 & Skip gram & 91.2 & 89.3 \\
\hline 200 & CBOW & 90.7 & 86.7 \\
\hline 200 & Skip gram & 90.3 & 89.5 \\
\hline 300 & CBOW & 90.6 & 88.6 \\
\hline 300 & Skip gram & 92.0 & 88.7 \\
\hline 400 & CBOW & 91.5 & 88.6 \\
\hline 400 & Skip gram & 90.2 & \\
\hline
\end{tabular}




\section{Table 7 (on next page)}

The Results of CNN-LSTM architecture 


\begin{tabular}{|c|c|c|c|}
\hline Number of feature vectors & Type of Word2vec model & Accuracy (Test) & $\begin{array}{c}\text { Accuracy (10-cross fold } \\
\text { validation) }\end{array}$ \\
\hline 100 & CBOW & 91.1 & 86.4 \\
\hline 100 & Skip gram & 90.9 & 87.9 \\
\hline 200 & CBOW & 91.8 & 88.8 \\
\hline 200 & Skip gram & 93.0 & 89.1 \\
\hline 300 & CBOW & 90.7 & 87.0 \\
\hline 300 & Skip gram & 92.2 & 87.3 \\
\hline 400 & CBOW & 91.5 & 89.2 \\
\hline 400 & Skip gram & 92.4 & \\
\hline
\end{tabular}




\section{Table 8 (on next page)}

The Results of the CNN-GRU model 


\begin{tabular}{|c|c|c|c|}
\hline Number of feature vectors & Type of Word2vec model & Accuracy (Test) & $\begin{array}{c}\text { Accuracy (10-cross fold } \\
\text { validation) }\end{array}$ \\
\hline 100 & CBOW & 91.7 & 84.7 \\
\hline 100 & Skip gram & 92.8 & 88.3 \\
\hline 200 & CBOW & 93.2 & 86.7 \\
\hline 200 & Skip gram & 92.9 & 89.5 \\
\hline 300 & CBOW & 91.4 & 87.2 \\
\hline 300 & Skip gram & 92.5 & 86.6 \\
\hline 400 & CBOW & 91.8 & 89.5 \\
\hline 400 & Skip gram & 92.6 & \\
\hline
\end{tabular}

